\title{
Beryllium monohydride (BeH): Where we are now, after 86 years of spectroscopy
}

\author{
Nikesh S. Dattani, ${ }^{1,2}$ ? \\ ${ }^{1}$ Physical and Theoretical Chemistry Laboratory, Department of Chemistry, \\ Oxford University, OX1 3QZ, Oxford, UK, and \\ ${ }^{2}$ Quantum Chemistry Laboratory, Department of Chemistry, Kyoto University, 606-8502, Kyoto, Japan,
}

\begin{abstract}
$\mathrm{BeH}$ is one of the most important benchmark systems for $a b$ initio methods and for studying Born-Oppenheimer breakdown. However the best empirical potential and best $a b$ initio potential for the ground electronic state to date give drastically different predictions in the long-range region beyond which measurements have been made, which is about $\sim 1000 \mathrm{~cm}^{-1}$ for ${ }^{9} \mathrm{BeH}, \sim 3000$ $\mathrm{cm}^{-1}$ for ${ }^{9} \mathrm{BeD}$, and $\sim 13000 \mathrm{~cm}^{-1}$ for ${ }^{9} \mathrm{BeT}$. Improved empirical potentials and Born-Oppenheimer breakdown corrections have now been built for the ground electronic states $X\left(1^{2} \Sigma^{+}\right)$of all three isotopologues. The predicted dissociation energy for ${ }^{9} \mathrm{BeH}$ from the new empirical potential is now closer to the current best $a b$ initio prediction by more than $66 \%$ of the discrepancy between the latter and the previous best empirical potential. The previous best empirical potential predicted the existence of unobserved vibrational levels for all three isotopologues, and the current best $a b$ initio study also predicted the existence of all of these levels, and four more. The present empirical potential agrees with the ab initio prediction of all of these extra levels not predicted by the earlier empirical potential. With one exception, all energy spacings between vibrational energy levels for which measurements have been made, are predicted with an agreement of better than $1 \mathrm{~cm}^{-1}$ between the new empirical potential and the current best ab initio potential, but some predictions for unobserved levels are still in great disagreement, and the equilibrium bond lengths are different by orders of magnitude.
\end{abstract}

PACS numbers: 02.60.Ed, 31.50.Bc , 82.80.-d , 31.15.ac, 33.20.-t, , 82.90.+j, 97, , 98.38.-j , 95.30.Ky

With only $5 \mathrm{e}^{-}, \mathrm{BeH}$ is the simplest neutral open shell molecule, and is therefore of paramount importance in benchmarking $a b$ initio methods. It has been the subject of a plethora of ab initio studies [1 24], with the first Hartree-Fock level endeavor being in 1967. It is also the second lightest neutral heteronuclear molecule after $\mathrm{LiH}$, and the only neutral diatomic for which spectroscopic measurements on a tritium isotopologue have been performed, making it currently the best benchmark system apart from $\mathrm{H}_{2}$ for studying the breakdown of the BornOppenheimer approximation [15, 21, 24, 26]. Due to its simplicity, BeH is expected to be present in astronomical contexts such as exoplanetary atmospheres, cool stars, and the interstellar medium [27], but in the context of astronomy, has only been found on our Sun, in the two studies described in [28, 29]. Finally, the extraordinarily long half-life of the halo nucleonic atom ${ }^{11} \mathrm{Be}$ makes ${ }^{11} \mathrm{BeH}$ a compelling candidate for the formation of the first halo nucleonic molecule [26].

Spectroscopic measurements on ${ }^{9} \mathrm{BeH}$ date back to 1928 30, 31, and on ${ }^{9} \mathrm{BeD}$ date back to 1935 32. By 1937 there was already an octad of publications on the molecule [30 37]. Since then, higher-resolution spectra have been measured for both of these isotopologues, and also for ${ }^{9} \mathrm{BeT}$ in 38 . Before the present paper, the most thorough empirical analysis of ${ }^{9} \mathrm{BeH},{ }^{9} \mathrm{BeD}$, and ${ }^{9} \mathrm{BeT}$ was that of [25], where empirical potentials were built for all three isotopologues, based on a fit to data from [25, 38, 41].

\footnotetext{
* dattani.nike@gmail.com
}

This state of the art 2006 empirical study left behind various mysteries which remained unsolved for the last 8 years:

1. The 2006 study predicted that the ${ }^{9} \mathrm{BeH}$ dissociation energy was $\mathfrak{D}_{e}=17590 \pm 200 \mathrm{~cm}^{-1}$ [25] which is higher than the value of $\mathfrak{D}_{e}=17426 \pm 100 \mathrm{~cm}^{-1}$ in the 1975 experimental study 43 by more than the latter's uncertainty. The most recent $a b$ initio study (published in 2011) of ${ }^{9} \mathrm{BeH}$ [24] predicted its dissociation energy to be $\mathfrak{D}_{e}=17702 \mathrm{~cm}^{-1}$ which is another $112 \mathrm{~cm}^{-1}$ higher than the 2006 value, and is higher than the 1975 value by almost three times the latter's estimated uncertainty.

2. The 2006 analysis was unable to determine a meaningful value for the leading term $u_{0}^{\mathrm{H}}$ of the adiabatic BOB (Born-Oppenheimer breakdown) correction function, which helps define the isotopologue shifts in $\mathfrak{D}_{e}$ and $r_{e}$.

3. The 2006 potentials predicted the existence of 1 more vibrational level for ${ }^{9} \mathrm{BeH}, 3$ more vibrational levels for ${ }^{9} \mathrm{BeD}$, and 15 more vibrational levels for ${ }^{9} \mathrm{BeT}$ than have ever been observed in experiments. The 2011 ab initio study then predicted the extra levels, but also one further vibrational level for ${ }^{9} \mathrm{BeH}, 2$ further levels for ${ }^{9} \mathrm{BeD}$, and 1 further vibrational level for ${ }^{9} \mathrm{BeT}(!)$.

As mentioned in the final section of the 2006 paper [25], the predictions of the unobserved vibrational levels in that study were questionable. In fact the large uncertainty in the predicted value of $\mathfrak{D}_{e}$, the inability to de- 
Figure 1. Comparison of the rotationless adiabatic potentials from 2014 [this work] and 2011 [24] for the ground state of ${ }^{9}$ BeH. Observed vibrational levels are blue and levels predicted by the 2014 potential are gray. The red curve represents the expected long-range behavior according to theory $\left(C_{m}\right.$ values are in Table $\square$ and damping functions $D_{m}(r)$ are the Douketis-type functions defined in 42 with $s=-2$ and $\left.\rho=0.9\right)$.

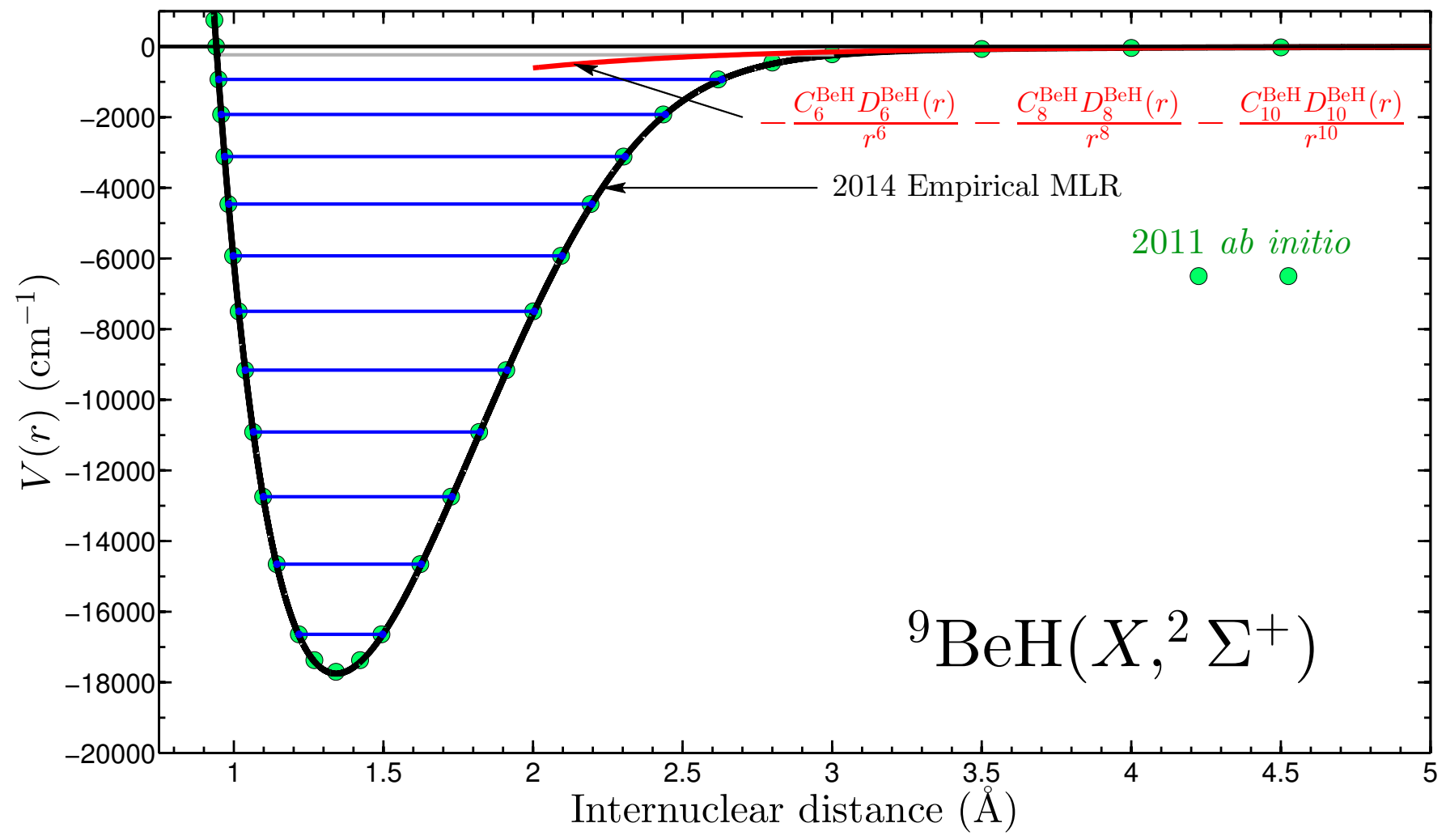

termine a meaningful value of $u_{0}^{\mathrm{H}}$, and the lack of confidence in the predictions of the unobserved vibrational levels were all the result of:

1. the fact that the highest vibrational level at which data were available, for any isotopologue, was $\sim$ $1000 \mathrm{~cm}^{-1}$ below the dissociation limit, and

2. the study used an EMO (expanded Morse oscillator) model for the potential, which dies off faster than exponentially with respect to the internuclear distance $r$, while theory dictates that for very large $r$, the potential should approach the dissociation asymptote with an inverse-power form (much more slowly than the EMO model does).

No new data has been published for any of the BeH isotopologues, so the $\sim 1000 \mathrm{~cm}^{-1}$ gap in spectroscopic guidance still presents a challenge. However, by using a model superior to the EMO we:

1. are able to bring the $\mathfrak{D}_{e}$ for $\mathrm{BeH}$ closer to the 2011 $a b$ initio value by more than $66 \%$ of the size of the discrepancy between the 2006 and 2011 values,

2. are able to determine $u_{0}^{\mathrm{H}}$ with an estimated uncertainty of $\pm 10 \mathrm{~cm}^{-1}$,
3. confirm that the extra predicted levels in the 2006 empirical analysis [25], and exactly the same number of further predicted levels as in the $2011 a b$ initio study [24] do in fact exist in our MLR potentials.

4. The present fit to the same spectroscopic data as in 2006, is also able to reproduce the measured energy transitions on average about $7 \%$ closer than the 2006 potential, with far fewer digits in its parametrization.

The 2006 study used the EMO model because there was reason to believe that the long-range tail of the rotationless potential had a barrier (see discussion and references in the fourth paragraph of this paper's Conclusions section). However, the 2011 ab initio calculation [24] has earned our trust (it predicted all but one of the ${ }^{9} \mathrm{BeH}$, ${ }^{9} \mathrm{BeD}$, and ${ }^{9} \mathrm{BeT}$ vibrational spacings in the data region to within $1 \mathrm{~cm}^{-1}$ of the spacings given by the $2006 \mathrm{em}-$ pirical potential), and it shows no signs of the existence of a rotationless barrier. Furthermore, the theoretical long-range potential based on the tremendously accurate long-range constants $\left(C_{6}, C_{8}\right.$, and $\left.C_{10}\right)$ which were recently calculated [4] for $\mathrm{BeH}$ (presented in Table I), also suggests that there is no barrier or turning points in the long-range.

This eliminates any doubt that there may have 
Table I. Long-range coefficients with and without finite-mass corrections for hydrogen. ${ }^{\infty} \mathrm{H}$ denotes the approximation where a hydrogen atom has an infinite atomic mass. All values are in atomic units. Numbers were generously provided by Professor Jim Mitroy of Charles Darwin University in (Australia) 44.

\begin{tabular}{ccccc} 
Atom 1 & Atom 2 & $C_{6}$ & $C_{8}$ & \multicolumn{1}{c}{$C_{10}$} \\
\hline $\mathrm{Be}$ & ${ }^{\infty} \mathrm{H}$ & 34.77918 & 1213.132 & 46593.36 \\
$\mathrm{Be}$ & $\mathrm{T}$ & 34.79610 & 1213.825 & 46624.91 \\
$\mathrm{Be}$ & $\mathrm{D}$ & 34.80452 & 1214.171 & 46640.62 \\
$\mathrm{Be}$ & $\mathrm{H}$ & 34.82984 & 1215.209 & 46687.87
\end{tabular}

been, that we should not use the more accurate MLR (Morse/Long-range) model for the potential, whose structure is very similar to the EMO for moderate values of the internuclear distance $r$, but unlike the EMO the correct long-range form of the potential according to theory, is promised by the mathematical structure of the MLR. In fact, in 2011 an MLR potential was fitted to spectroscopic data for the $c\left(1^{3} \Sigma_{g}^{+}\right)$-state of ${ }^{6,6} \mathrm{Li}_{2}$ and ${ }^{7,7} \mathrm{Li}_{2}$, where there was a gap of more than $5000 \mathrm{~cm}^{-1}$ between data near the bottom of the potential's well, and data at the very top 45. In 2013 spectroscopic measurements were made in the very middle of this gap, and it was found that the vibrational energies predicted by the MLR potential from 45 were correct to about $1 \mathrm{~cm}^{-1}$ [46]. This means that the MLR model can be capable of making very accurate predictions outside of the data range, which is particularly pertinent for the present case of BeH which at the moment still suffers from the issues mentioned above.

\section{THE NEW POTENTIAL}

With the exception of using an improved model for the $\mathrm{BeH}$ adiabatic potential energy function $V_{\text {ad }}^{(1)}(r)$ and the BOB correction functions $\Delta V_{\text {ad }}^{(\alpha)}$ and $g^{(\alpha)}(r)$, we fit the same Hamiltonian to the same data set as used in the 2006 empirical analysis of $\mathrm{BeH}$ [25]. Instead of using the EMO model of the 2006 study, we use the MLR model for $V_{\mathrm{ad}}^{(1)}(r)$, exactly as described for the $a$-state of $\mathrm{Li}_{2}$ in 2011[45]. We also use the exact same definitions of $\Delta V_{\mathrm{ad}}^{(\alpha)}$ and $g^{(\alpha)}(r)$ as in that $\mathrm{Li}_{2}$ study [45; the only difference from those used in the $2006 \mathrm{BeH}$ study is the use of two separate Surkus powers in $\Delta V_{\text {ad }}^{(\alpha)}: p_{\text {ad }}$ and $q_{\text {ad }}$, rather than just $p_{\text {ad }}$.

For each reference isotopologue, the long-range coefficients were taken from Table I] Relativistic effects were not taken into account in the calculation of these long-range coefficients because the relativistic correction for hydrogen is expected to be about 10 times smaller than the finite-mass correction [44], and the overall error due to neglecting relativistic effects was expected to be smaller than the error due to the Be structure model [44].
Table II. Parameters defining the recommended MLR potential for the $X\left({ }^{2} \Sigma^{+}\right)$-state of the reference isotopologue ${ }^{9} \mathrm{BeH}$ and the BOB correction functions for all other isotopologues with ${ }^{9} \mathrm{Be}$. Parameters in square brackets were held fixed in the fit, while numbers in round brackets are $95 \%$ confidence limit uncertainties in the last digit(s) shown. The potential also incorporates damping functions according to 47, with $s=-2$ and $\rho=0.9$.

\begin{tabular}{llllll}
\hline \hline & $\operatorname{MLR}_{5,3}^{2.35}(6)$ & & $\operatorname{BOB}_{6,4}^{\mathrm{ad}}(4)$ & \multicolumn{2}{l}{$\operatorname{BOB}_{3,3}^{\mathrm{na}}(4,2)$} \\
\hline $\mathfrak{D}_{e}$ & $17664(200) \mathrm{cm}^{-1}$ & $u_{0}^{\mathrm{H}}$ & $-13(10) \mathrm{cm}^{-1}$ & $t_{0}^{\mathrm{Be}}$ & {$[0]$} \\
$r_{e}$ & $1.342396(2) \AA$ & $u_{1}^{\mathrm{H}}$ & $85.07 \mathrm{~cm}^{-1}$ & $t_{1}^{\mathrm{Be}}$ & $-6.1 \times 10^{-4}$ \\
$C_{6}$ & {$[34.82984]$ a.u. } & $u_{2}^{\mathrm{H}}$ & $23.51 \mathrm{~cm}^{-1}$ & $t_{2}^{\mathrm{Be}}$ & $5.87 \times 10^{-3}$ \\
$C_{8}$ & {$[1215.209]$ a.u. } & $u_{3}^{\mathrm{H}}$ & $51.10 \mathrm{~cm}^{-1}$ & $t_{3}^{\mathrm{Be}}$ & $-1.2 \times 10^{-4}$ \\
$C_{10}$ & {$[46687.87]$ a.u. } & $u_{4}^{\mathrm{H}}$ & $75.10 \mathrm{~cm}^{-1}$ & $t_{4}^{\mathrm{Be}}$ & $1.05 \times 10^{-2}$ \\
$\beta_{0}$ & 1.21862088 & $u_{\infty}^{\mathrm{H}}$ & {$[0] \mathrm{cm}^{-1}$} & $t_{\infty}^{\mathrm{Be}}$ & {$[0]$} \\
$\beta_{1}$ & 1.9636945 & & & & \\
$\beta_{2}$ & 7.598094 & & & $t_{0}^{\mathrm{H}}$ & {$[0]$} \\
$\beta_{3}$ & 14.16754 & & & $t_{1}^{\mathrm{H}}$ & $1.929 \times 10^{-3}$ \\
$\beta_{4}$ & 15.2569 & & & $t_{2}^{\mathrm{H}}$ & $-6.21 \times 10^{-3}$ \\
$\beta_{5}$ & 9.2876 & & & $t_{\infty}^{\mathrm{H}}$ & {$[0]$} \\
$\beta_{6}$ & 2.52 & & & & \\
& & & & &
\end{tabular}

Likewise, finite mass corrections for ${ }^{9} \mathrm{Be}$ were smaller than the estimated uncertainty in the overall values of the long-range coefficients 444. While this was also true for the isotopes of hydrogen, the differences across the three isotopes are expected to be reliable [44.

The final MLR potential and BOB correction function parameters are presented in Table II.

\section{COMPARISON WITH 2006 EMPIRICAL AND 2011 AB INITIO POTENTIALS}

In Figs 1, 2, 3 and 4 the final adiabatic potentials for all three isotopologues are compared to the $2006 \mathrm{EMO}$ model results and to the $2011 a b$ initio results. While $a b$ initio potentials with varying amounts of included theory were discussed in the original paper [24], all figures, tables and discussion about rotationless potentials in this paper refer to the final adiabatic potentials for each isotopologue, referred in the original paper as " $\mathrm{CV}+\mathrm{F}+\mathrm{R}+\mathrm{D}$ " to indicate the use of MR-ACPF/aug-cc-pCV7Z(i) (denoted by $\mathrm{CV}$ ), an estimate of electron correlation effects beyond the approximations of MR-ACPF (denoted by F), second-order Douglas-Kroll-Hess (DKH) scalar relativistic corrections (denoted by $\mathrm{R}$ ) and mass-dependent diagonal BOB corrections (denoted by D). Furthermore, the 2011 ab inito study of 24 also included non-adiabatic BOB corrections for the calculation of rotational constants $B_{v}$ and centrifugal distortion constants $D_{v}$. The values of these constants with the non-adiabatic corrections taken into account were not presented in the original paper [24], but were generously provided by the author for the present comparison.

No point-wise representation for these final $(\mathrm{CV}+\mathrm{F}+$ $\mathrm{R}+\mathrm{D})$ potentials were listed in the original paper [24], 
Table III. Comparison of spectroscopic constants derived from the three studies. Differences denoted by the columns with a $\Delta$ in the heading are calculated by subtracting the value in question from the respective 2014 value. The second row of the header lists the units for each column's quantities.

\begin{tabular}{|c|c|c|c|c|c|c|c|c|}
\hline & $\begin{array}{c}r_{e} \\
{[\mathrm{pm}]}\end{array}$ & $\begin{array}{l}\Delta r_{e} \\
{[\mathrm{am}]}\end{array}$ & $\begin{array}{c}\mathfrak{D}_{e} \\
{\left[\mathrm{~cm}^{-1}\right]}\end{array}$ & $\begin{array}{c}\Delta \mathfrak{D}_{e} \\
{\left[\mathrm{~cm}^{-1}\right]}\end{array}$ & $\begin{array}{c}B_{0} \\
{\left[\mathrm{~cm}^{-1}\right]}\end{array}$ & $\begin{array}{c}\Delta B_{0} \\
{\left[\mathrm{~cm}^{-1}\right]}\end{array}$ & $\begin{array}{c}D_{0} \times 10^{-3} \\
{\left[\mathrm{~cm}^{-1}\right]}\end{array}$ & $\begin{array}{c}\Delta D_{0} \times 10^{-3} \\
{\left[\mathrm{~cm}^{-1}\right]}\end{array}$ \\
\hline \multicolumn{9}{|c|}{${ }^{9} \mathrm{BeH}$} \\
\hline 2014 Empirical & $134.2396(2)$ & - & $17664(200)$ & - & 10.16563 & - & 1.0267 & - \\
\hline $2011 a b$ initio 24 & 134.144 & 95600 & 17702 & 38 & 10.1650 & -0.00063 & 1.0272 & -0.0005 \\
\hline 2006 Empirical 25] & $134.23940(12)$ & 200 & $17590(200)$ & 74 & 10.16571 & -0.00008 & 1.0272 & -0.0005 \\
\hline \multicolumn{9}{|c|}{${ }^{9} \mathrm{BeD}$} \\
\hline 2014 Empirical & $134.1728(2)$ & - & $17759(200)$ & - & 5.6253 & - & 0.3130 & - \\
\hline $2011 a b$ initio 24 & 134.124 & 48800 & 17711 & 48 & 5.6260 & 0.0007 & 0.3130 & 0.0000 \\
\hline 2006 Empirical 25 & $134.17197(12)$ & 8300 & 17597 & 161 & 5.6253 & 0.0000 & 0.3128 & 0.0002 \\
\hline \multicolumn{9}{|c|}{${ }^{9} \mathrm{BeT}$} \\
\hline 2014 Empirical & $134.1504(2)$ & - & $17762(200)$ & - & 4.1061 & - & 0.1664 & - \\
\hline 2011 ab initio 24 & 134.117 & 33400 & 17715 & 47 & 4.1067 & -0.0006 & 0.1664 & 0.0000 \\
\hline 2006 Empirical 25] & $134.14951(12)$ & 8900 & 17599 & 163 & 4.1060 & -0.0001 & 0.1663 & 0.0001 \\
\hline
\end{tabular}

but they were generously provided by the author for the present paper. The point-wise representation for each isotopologue listed the $(\mathrm{CV}+\mathrm{F}+\mathrm{R}+\mathrm{D})$ potential at 48 different internuclear distances, which when all plotted in Figs 1, 2, 3 and 4, proved to make the figures messy. Therefore these points were only plotted for fairly large values of $r$, and for small values of $r$, cubic splines were created through the dense meshes of $a b$ initio points, and points were only plotted from the interpolant at certain places chosen to maximize readability.

Table III compares spectroscopic constants derived from the present study to those derived from the 2006 25 and 2011 24 studies, and Table IV compares the predicted vibrational binding energies, zero point energies, and vibrational spacings. A similar comparison was made across Tables IV, V and VI of [24]. While those tables did not show $v=12$ for ${ }^{9} \mathrm{BeH}$ or $v=17$ for ${ }^{9} \mathrm{BeD}$ for the 2011 ab initio potentials and $v=13-18$ for ${ }^{9} \mathrm{BeT}$ for the 2006 empirical potential, those potentials did in fact support these levels, so their predictions are included in Table IV of the present paper.

In last column of Table IV it is emphasized that the disagreement between the present empirical potential and the $2011 a b$ initio potential of [24] only rises above 1 $\mathrm{cm}^{-1}$ for one vibrational spacing in the data region (the space between $v=9$ and $v=10$ for ${ }^{9} \mathrm{BeH}$ ). This was also the only vibrational spacing in the data region for which the disagreement between the predicted energy spacing of the $2011 a b$ initio and the 2006 empirical potential was larger than $1 \mathrm{~cm}^{-1}$. However, in the region where measurements have not been made, the $a b$ initio potential is for the most part in much better agreement with the present empirical potential than the 2006 empirical potential, particularly in the number of vibrational levels supported and in the energy spacings of higher vibrational energies.

Furthermore, in Table III we see that the 2011 ab initio potentials of [24] predicted a dissociation energy $\mathfrak{D}_{e}$ that is $74 \mathrm{~cm}^{-1}$ closer to the present empirical potential than the the $\mathfrak{D}_{e}$ predicted by the 2006 empirical potential of [25]. Combining this observation with those in the above paragraph, and the comparison of the potential energy curves in Figs 1, 2, 3 and 4, tells us that the $2011 a b$ initio potential actually made more accurate predictions than the 2006 empirical potential for various rotationless properties such as the dissociation energy, vibrational energy spacings for very high values of $v$, number of predicted levels, and the potential energy curves themselves are simply in better agreement.

Finally it is interesting to note that the predicted value for the leading term of the adiabatic BOB correction function for hydrogen $\left(u_{0}^{\mathrm{H}}=-13 \pm 10 \mathrm{~cm}^{-1}\right.$ in Table II), despite its large uncertainty due to the large gap in data between the highest observed vibrational level and the dissociation asymptote, is very similar to the value $\left(u_{0}^{\mathrm{H}}=-15.183 \pm 0.61 \mathrm{~cm}^{-1}\right)$ obtained for the recent extensive study on the ground $\left(X, 1^{2} \Sigma^{+}\right)$-state of $\mathrm{MgH}$, for which the fit was to 20103 spectroscopic data across $\mathrm{MgH}$ and $\mathrm{MgD}$ ( $c$.f. to the present study which involves a fit to 2821 data across $\mathrm{BeH}, \mathrm{BeD}$ and BeT). 


\section{CONCLUSION}

Spectroscopic studies of BeH date back to as early as 1928 [30, 31, 48, and Hartree-Fock level ab initio studies on the system go back as far as 1967 [1]. At present, about $11 \%, 17 \%$, and $21 \%$ of the adiabatic rotationless potentials for ${ }^{9} \mathrm{BeH},{ }^{9} \mathrm{BeD}$ and ${ }^{9} \mathrm{BeT}$ respectively, have not yet been covered by spectroscopic measurements. Consequently, the dissociation energy $\mathfrak{D}_{e}$, the number of vibrational levels, and the leading term of hydrogen's adiabatic BOB correction function $u_{0}^{\mathrm{H}}$ have been elusive, the latter having its first empirical value reported in this present work, and the former two varying greatly in the most recent empirical [25] and ab initio [24] studies before this work.

For all three isotopologues, the confidence intervals for the empirical values of the $\mathfrak{D}_{e}$ 's deduced from the present study are still about $\pm 200 \mathrm{~cm}^{-1}$ which is rather large for a $\mathfrak{D}_{e}$, especially for such a fundamental system. However, these empirical values are closer than ever to the state of the art $a b$ initio values [24] which differ by at most 48 $\mathrm{cm}^{-1}$ (in the case of ${ }^{9} \mathrm{BeD}$ ).

The present study predicts the exact same number of vibrational levels for all three isotopologues as the state of the art $a b$ initio study 24. Of the predicted levels, $v=11-12$ for ${ }^{9} \mathrm{BeH}, v=13-17$ for ${ }^{9} \mathrm{BeD}$, and $v=4-19$ for ${ }^{9} \mathrm{BeT}$ have never been observed experimentally. Of these unobserved transitions, $v=11$ for ${ }^{9} \mathrm{BeH}$, $v=13-15$ for ${ }^{9} \mathrm{BeD}$, and $v=4-18$ for ${ }^{9} \mathrm{BeT}$ are predicted to be bound by over $100 \mathrm{~cm}^{-1}$, by both the present study and the state of the art $a b$ initio study of [24], so they are likely to exist. The higher levels are in a region where the $a b$ initio vibrational energies had already started to deviate rather greatly from the present empirical potential, so confirmation of their existence awaits further experiments or higher level $a b$ initio calculations, perhaps with non-adiabatic BOB corrections included.

Finally, the nature of the long-range tail of $\mathrm{BeH}$ potentials has been an intriguing mystery for several decades. Early electronic structure studies [7, 8, 39] discussed the possibility of there being a rotationless barrier or multiple inflection points in the long-range region. This was at focus in subsequent $a b$ initio studies which found that these irregularities disappeared as the basis set size increased or other improvements were made [9, 10, 12, and even in later studies which again found such irregularities in the $r=3-5 \AA$ region [14, 17, 18, 49]. Figs 2. 3 and 4 provide a compelling reason to believe that no such irregularities found in earlier ab initio studies are genuine. The latest $a b$ initio study of [24], which in the data region agrees very well with experiments, did not show evidence of such irregularities. Since the figures show that no spectroscopic data is available in the $r=3-5 \AA$ region, the fact that the empirical potentials from the present analysis also do not appear to have such irregularities is a consequence of the fact that the model upon which they were based was designed to smoothly transition between Morse-like short-range to mid-range behavior, and inverse-power long-range behavior according to theory. Since the coefficients in Table $\mathrm{I}$ are all positive, the theoretical inverse-power tail of the long-range potential is monotonically decreasing with respect to $r$ and therefore cannot have a barrier or inflection point. Due to the large gap between the highest experimentally observed vibrational levels and the point at which the empirical MLR potentials join the theoretical long-range potentials (that we see in the figures), there may be some room for inflections which the MLR model was not able to capture, but the acute agreement with the accurate $a b$ initio potential energy curves rules out the possibility of a barrier and casts doubt on the existence of inflection points.

Finally, the new adiabatic potentials and BOB corrections functions for ${ }^{9} \mathrm{BeH},{ }^{9} \mathrm{BeD}$, and ${ }^{9} \mathrm{BeT}$ presented in this paper can be used to benchmark ab initio methods, especially for open shell molecules, since $\mathrm{BeH}$ is the simplest neutral open shell molecule with a stable ground electronic state. All of them are provided for a dense grid of internuclear distance values in a text file in this paper's Supplementary Material, along with MATLAB and FORTRAN programs to generate them at any internuclear distance using their analytic expressions. The present study adds BeH to the ever-growing list of molecules for which accurate empirical MLR-type potentials are available 45-47, 50, 70.

\section{NOTE ABOUT REFERENCES}

While 24 ab initio studies were referenced in the introductory paragraph to this paper, other references may have been missed due to the author not being aware of their existence. Likewise, experimental studies before 1937 and after 1974 have been cited, but other studies about which the author is unaware may exist. If the reader is aware of any such references, they are keenly encouraged to inform the author at dattani.nike@gmail.com.

\section{ACKNOWLEDGMENTS}

It is with pleasure that the author thanks Bob Le Roy of University of Waterloo (Canada) for suggesting $\mathrm{BeH}$ as an interesting molecule for testing extrapolation with analytic models. The author also gratefully thanks Jim Mitroy of Charles Darwin University (Australia) for providing his unpublished calculated values for the constants appearing in TableI and used for the models to which the data was fitted, Jacek Koput of Adam Mickiewicz University (Poland) for his very prompt supply of his pointwise $a b$ initio potentials for ${ }^{9} \mathrm{BeH},{ }^{9} \mathrm{BeD}$, and ${ }^{9} \mathrm{BeT}$ from [24, Yoshitaka Tanimura of Kyoto University (Japan) for his generous hospitality, and last but indubitably not least, Staszek Welsh of Oxford University (UK) for assistance with running many fits at a preliminary stage of this 
project. Financial support was generously provided by
JSPS.
[1] P. E. Cade and W. M. Huo, The Journal of Chemical Physics 47, 614 (1967)

[2] A. C. H. Chan, The Journal of Chemical Physics 49, 727 (1968)

[3] C. Bender and E. Davidson, Physical Review 183, 23 (1969).

[4] M. Jungen and R. Ahlrichs, Theoretica Chimica Acta 17, 339 (1970)

[5] R. S. Mulliken, International Journal of Quantum Chemistry 5, 95 (1971)

[6] H. E. Popkie, The Journal of Chemical Physics 54, 4597 (1971)

[7] P. S. Bagus, The Journal of Chemical Physics 58, 1886 (1973)

[8] J. Gerratt and M. Raimondi, Proceedings of the Royal Society A: Mathematical, Physical and Engineering Sciences 371, 525 (1980).

[9] D. L. Cooper, The Journal of Chemical Physics 80, 1961 (1984)

[10] M. Larsson, The Journal of Chemical Physics 81, 6409 (1984)

[11] M. Larsson, Physica Scripta 32, 97 (1985)

[12] C. Henriet and G. Verhaegen, Physica Scripta 33, 299 (1986)

[13] I. D. Petsalakis, G. Theodorakopoulos, and C. A. Nicolaides, The Journal of Chemical Physics 97, 7623 (1992).

[14] X. Li and J. Paldus, The Journal of Chemical Physics 102, $2013(1995)$

[15] J. M. Martin, Chemical Physics Letters 283, 283 (1998)

[16] F. Machado, O. Roberto-Neto, and F. Ornellas, Chemical Physics Letters 284, 293 (1998)

[17] I. D. Petsalakis, D. Papadopoulos, G. Theodorakopoulos, and R. J. Buenker, Journal of Physics B: Atomic, Molecular and Optical Physics 32, 3225 (1999)

[18] H. Meiß ner and J. Paldus, The Journal of Chemical Physics 113, 2622 (2000)

[19] M. P. Fülscher and L. Serrano-Andrés, Molecular Physics 100, 903 (2002)

[20] P. J. Bruna and F. Grein, Physical Chemistry Chemical Physics 5, 3140 (2003).

[21] S. Bubin and L. Adamowicz, The Journal of Chemical Physics 126, 214305 (2007).

[22] J. Pitarch-Ruiz, J. Sánchez-Marin, A. M. Velasco, and I. Martin, The Journal of Chemical Physics 129, 054310 (2008)

[23] J. Pitarch-Ruiz, J. Sánchez-Marín, and A. M. Velasco, Journal of Computational Chemistry 29, 523 (2008).

[24] J. Koput, The Journal of chemical physics 135, 244308 (2011)

[25] R. J. Le Roy, D. R. Appadoo, R. Colin, and P. F. Bernath, Journal of Molecular Spectroscopy 236, 178 (2006)

[26] N. S. Dattani and S. Welsh, in 69th International Symposium on Molecular Spectroscopy, Vol. RD01 (2014) p. RD01.

[27] B. Yadin, T. Veness, P. Conti, C. Hill, S. N. Yurchenko, and J. Tennyson, Monthly Notices of the Royal Astronomical Society 425, 34 (2012).
[28] H. Wohl, Solar Physics 16, 362 (1971).

[29] R. Shanmugavel, S. Bagare, N. Rajamanickam, and B. K. Kumar, Serbian Astronomical Journal , 51 (2008)

[30] W. W. Watson, Physical Review 32, 600 (1928).

[31] M. Peterson, Physical Review 31 (1928).

[32] P. G. Koontz, Physical Review 48, 707 (1935).

[33] W. W. Watson, Physical Review 34, 372 (1929).

[34] W. W. Watson and A. Parker, Physical Review 36, 1019 (1930).

[35] W. W. Watson, Physical Review 37, 167 (1931).

[36] E. Olsson, Zeitschrift für Physik 73, 732 (1932).

[37] W. W. Watson and R. F. Humphreys, Physical Review $\mathbf{5 2}, 318$ (1937).

[38] D. De Greef and R. Colin, Journal of Molecular Spectroscopy 53, 455 (1974).

[39] R. Colin, C. Drèze, and M. Steinhauer, Canadian Journal of Physics 61, 641 (1983).

[40] C. Focsa, P. Bernath, R. Mitzner, and R. Colin, Journal of Molecular Spectroscopy 192, 348 (1998).

[41] A. Shayesteh, K. Tereszchuk, P. F. Bernath, and R. Colin, The Journal of Chemical Physics 118, 1158 (2003).

[42] R. J. Le Roy, C. C. Haugen, J. Tao, and H. Li, Molecular Physics 109, 435 (2011).

[43] R. Colin and D. De Greef, Canadian Journal of Physics 53, 2142 (1975).

[44] J. Mitroy, Private Communication (2014).

[45] N. S. Dattani and R. J. Le Roy, Journal of Molecular Spectroscopy 268, 199 (2011)

[46 M. Semczuk, X. Li, W. Gunton, M. Haw, N. S. Dattani, J. Witz, A. K. Mills, D. J. Jones, and K. W. Madison, Physical Review A 87, 052505 (2013)

[47] R. J. Le Roy, C. C. Haugen, J. Tao, and H. Li, Molecular Physics 109, 435 (2011).

[48] E. Wigner and E. E. Witmer, Zeitschrift für Physik 51, 859 (1928)

[49] X. Li and J. Paldus, Private Communication (2005).

[50] R. J. Le Roy, Y. Huang, and C. Jary, The Journal of Chemical Physics 125, 164310 (2006)

[51] R. J. L. Roy and R. D. E. Henderson, Molecular Physics 105, $663(2007)$

[52] H. Salami, A. J. Ross, P. Crozet, W. Jastrzebski, P. Kowalczyk, and R. J. Le Roy, The Journal of Chemical Physics 126, 194313 (2007)

[53] A. Shayesteh, R. D. E. Henderson, R. J. Le Roy, and P. F. Bernath, The Journal of Physical Chemistry. A 111, 12495 (2007)

[54] H. Li and R. J. Le Roy, Physical Chemistry Chemical Physics : PCCP 10, 4128 (2008).

[55] R. J. Le Roy, N. S. Dattani, J. a. Coxon, A. J. Ross, P. Crozet, and C. Linton, The Journal of Chemical Physics 131, 204309 (2009).

[56] J. A. Coxon and P. G. Hajigeorgiou, The Journal of Chemical Physics 132, 094105 (2010)

[57] A. Stein, H. Knöckel, and E. Tiemann, The European Physical Journal D 57, 171 (2010)

[58] H. Li, P.-N. Roy, and R. J. Le Roy, The Journal of Chemical Physics 133, 104305 (2010) 
Figure 2. Comparison of the rotationless adiabatic potentials from 2014 [this work] and 2011 [24] for the ground state of ${ }^{9} \mathbf{B e H}$. Observed vibrational levels are blue and levels predicted by the 2014 potential are gray. The red curve represents the expected long-range behavior according to theory $\left(C_{m}\right.$ values are in Table $\square$ and damping functions $D_{m}(r)$ are the Douketis-type functions defined in 42 with $s=-2$ and $\left.\rho=0.9\right)$.

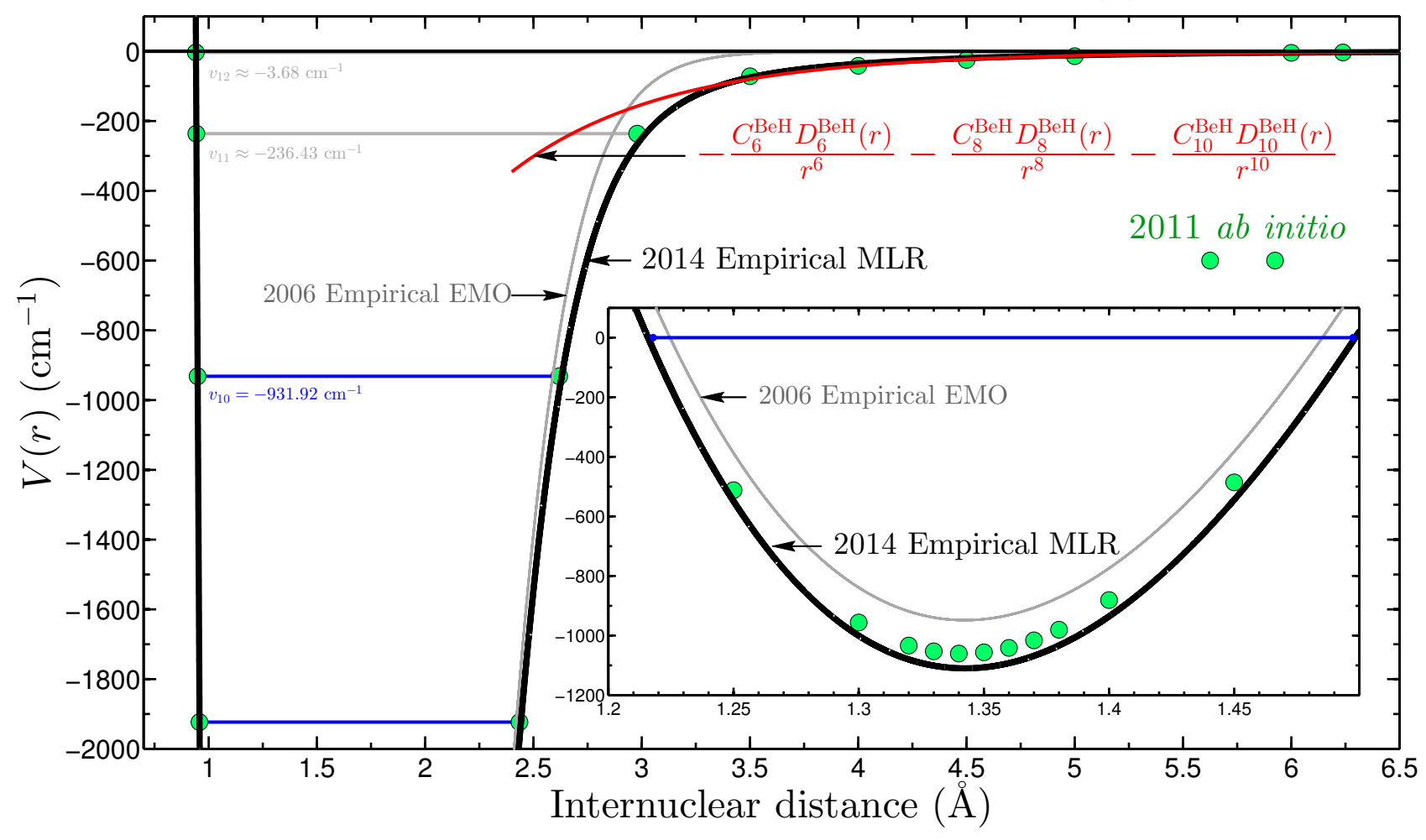

Figure 3. Comparison of the rotationless adiabatic potentials from 2014 [this work] and 2011 [24] for the ground state of ${ }^{9}$ BeD. Observed vibrational levels are blue and levels predicted by the 2014 potential are gray. The red curve represents the expected long-range behavior according to theory $\left(C_{m}\right.$ values are in Table I and damping functions $D_{m}(r)$ are the Douketis-type functions defined in 42 with $s=-2$ and $\left.\rho=0.9\right)$.

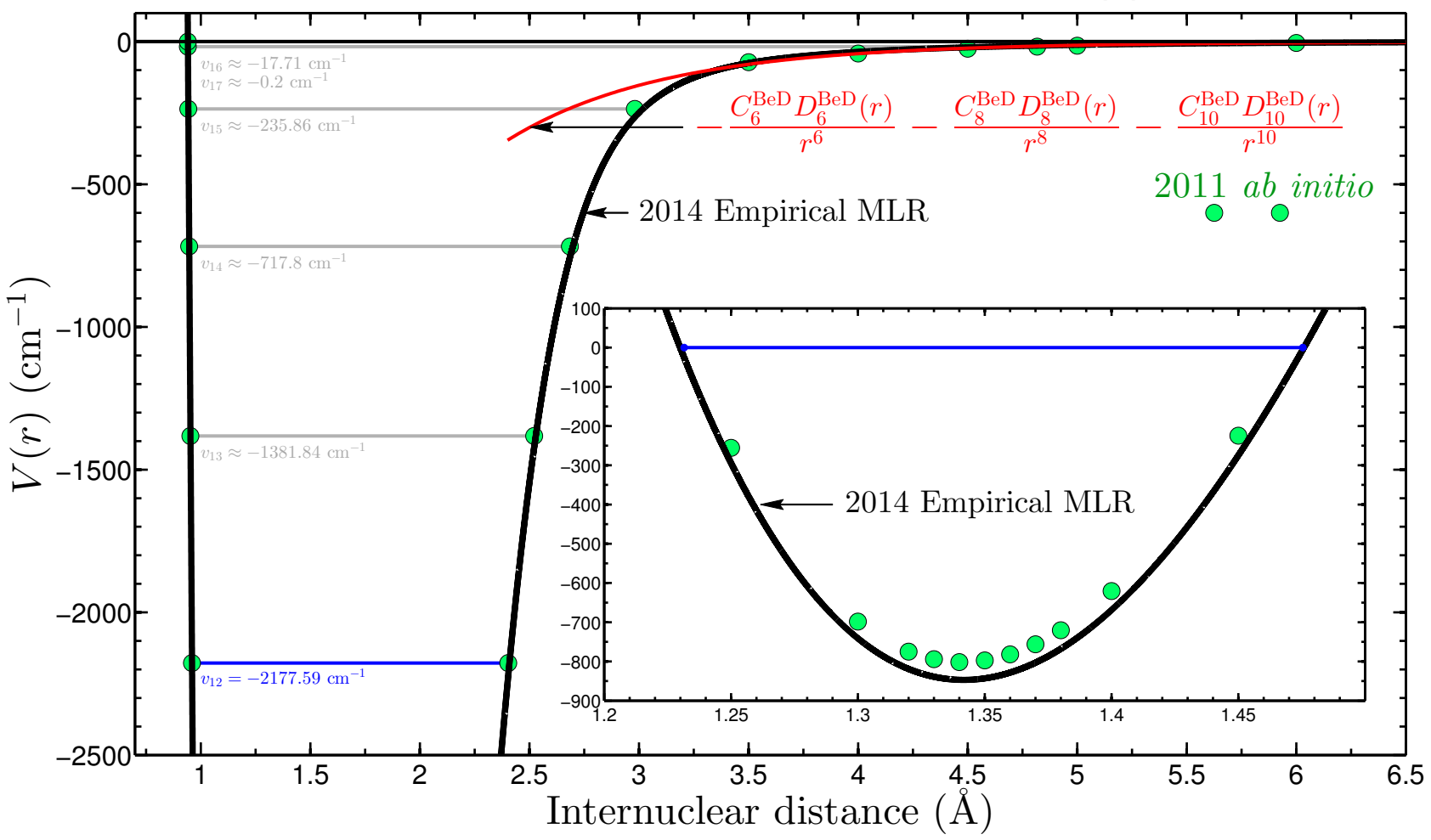


Figure 4. Comparison of the rotationless adiabatic potentials from 2014 [this work] and 201124 for the ground state of ${ }^{9}$ BeT. Observed vibrational levels are blue and levels predicted by the 2014 potential are gray. The red curve represents the expected long-range behavior according to theory $\left(C_{m}\right.$ values are in Table $\square$ and damping functions $D_{m}(r)$ are the Douketis-type functions defined in 42 with $s=-2$ and $\left.\rho=0.9\right)$.

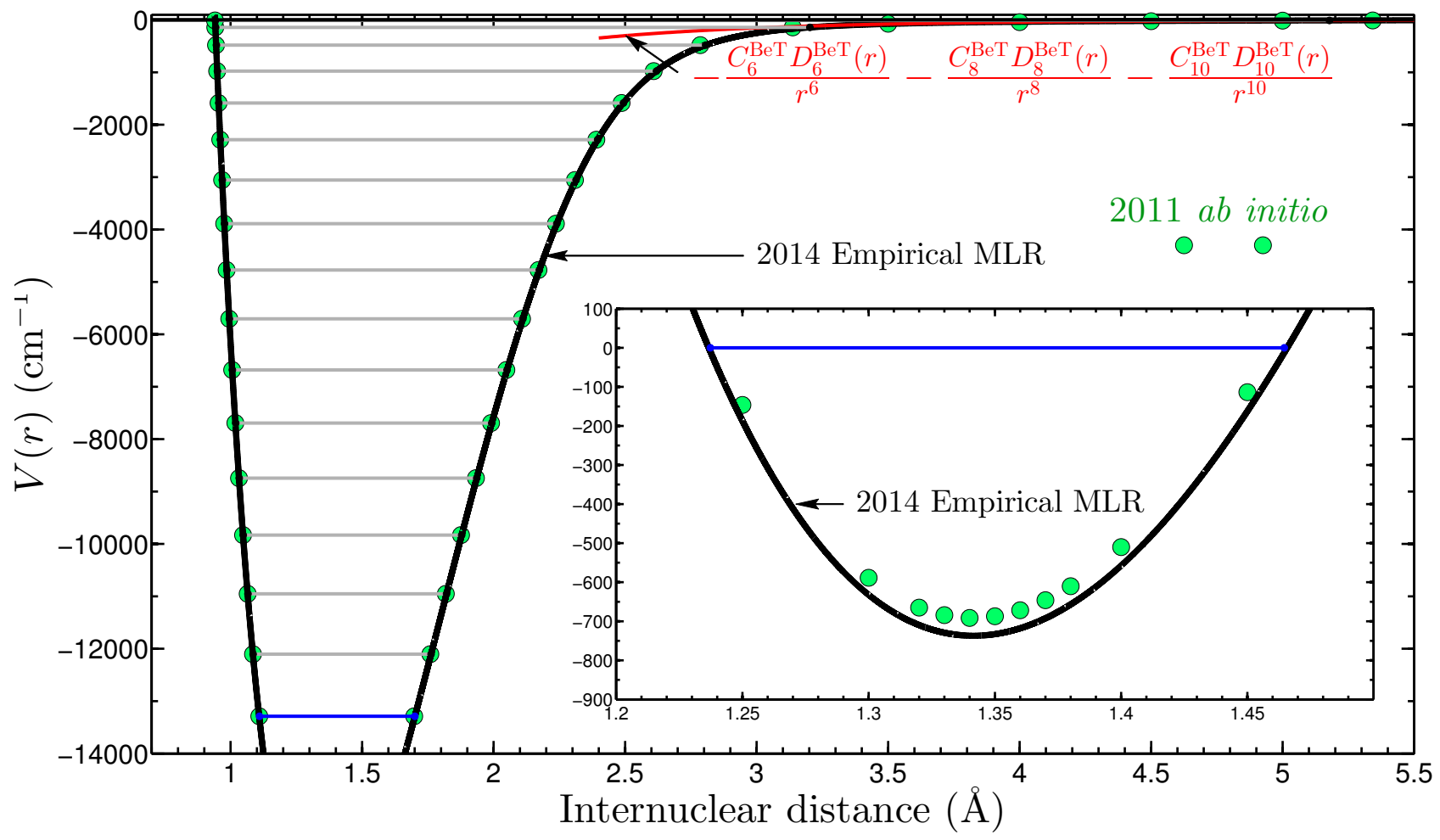

[59] L. Piticco, F. Merkt, A. A. Cholewinski, F. R. McCourt, and R. J. Le Roy, Journal of Molecular Spectroscopy 264, 83 (2010)

[60] M. Ivanova, A. Stein, A. Pashov, A. V. Stolyarov, H. Knöckel, and E. Tiemann, The Journal of Chemical Physics 135, 174303 (2011)

61| F. Xie, L. Li, D. Li, V. B. Sovkov, K. V. Minaev, V. S. Ivanov, A. M. Lyyra, and S. Magnier, The Journal of Chemical Physics 135, 024303 (2011)

[62] T. Yukiya, N. Nishimiya, Y. Samejima, K. Yamaguchi, M. Suzuki, C. D. Boone, I. Ozier, and R. J. Le Roy, Journal of Molecular Spectroscopy 283, 32 (2013).

[63] H. Knöckel, S. Rühmann, and E. Tiemann, The Journal of Chemical Physics 138, 094303 (2013).

[64] Y. Tritzant-Martinez, T. Zeng, A. Broom, E. Meiering, R. J. Le Roy, and P.-N. Roy, The Journal of Chemical
Physics 138, 234103 (2013).

[65] L. Wang, D. Xie, R. J. Le Roy, and P.-N. Roy, The Journal of Chemical Physics 139, 034312 (2013).

[66] H. Li, X.-L. Zhang, R. J. Le Roy, and P.-N. Roy, The Journal of Chemical Physics 139, 164315 (2013).

[67] G. Li, I. E. Gordon, P. G. Hajigeorgiou, J. A. Coxon, and L. S. Rothman, Journal of Quantitative Spectroscopy and Radiative Transfer 130, 284 (2013)

[68] W. Gunton, M. Semczuk, N. Dattani, and K. Madison, Physical Review A 88, 062510 (2013)

[69] V. V. Meshkov, A. V. Stolyarov, M. C. Heaven, C. Haugen, and R. J. LeRoy, The Journal of Chemical Physics 140, 064315 (2014)

[70] N. S. Dattani, L. N. Zack, M. Sun, E. R. Johnson, R. J. Le Roy, and L. M. Ziurys, physics.chem-ph , arXiv:1408.2276 (2014). 
Table IV. Comparison of the binding energies, denoted $G\left(v_{i}\right)$; zero-point energies (ZPE); and vibrational energy spacings, denoted $\omega_{i} \equiv G\left(v_{i}\right)-$ $G\left(v_{i-1}\right)$; for all three isotopologues. The last column is the difference between the two columns directly prior. Discrepancies of $\geq 1$ cm ${ }^{-1}$ are marked by one star for spacings outside the data range, and by three stars when the discrepancy was this large for a spacing within the data range. Lines with black font indicate levels that have been observed experimentally and lines with bold blue font are for unobserved levels that have only been predicted.

\begin{tabular}{|c|c|c|c|c|c|c|c|}
\hline$v$ & $\begin{array}{c}2006 \text { Empirical } \\
25\end{array}$ & $\begin{array}{c}2011 \text { ab initio } \\
24\end{array}$ & $\begin{array}{r}2014 \text { Empirical } \\
\text { [Present work] }\end{array}$ & $\begin{array}{c}2006 \text { Empirical } \\
25\end{array}$ & $\begin{array}{c}2011 \text { ab initio } \\
24\end{array}$ & $\begin{array}{r}2014 \text { Empirical } \\
\text { [Present work] }\end{array}$ & $\begin{array}{c}\Delta \\
{[\omega(2014)-\omega(2011)]}\end{array}$ \\
\hline
\end{tabular}

\begin{tabular}{|c|c|c|c|c|c|c|c|c|c|}
\hline \multicolumn{10}{|c|}{${ }^{9} \mathrm{BeH}$} \\
\hline 0 & -16567.7651 & -16679.67 & -16641.7725 & $\mathrm{ZPE}$ & 1021.290 & 1022.33 & & & \\
\hline 1 & -14581.3475 & -14693.12 & -14655.3569 & $\omega_{1}$ & 1986.4176 & 1986.55 & 1986.4156 & -0.13 & \\
\hline 2 & -12670.8937 & -12782.57 & -12744.9033 & $\omega_{2}$ & 1910.4538 & 1910.55 & 1910.4536 & -0.10 & \\
\hline 3 & -10838.5033 & -10950.10 & -10912.5124 & $\omega_{3}$ & 1832.3904 & 1832.47 & 1832.3909 & -0.08 & \\
\hline 4 & -9087.4251 & -9198.99 & -9161.4384 & $\omega_{4}$ & 1751.0782 & 1751.11 & 1751.074 & -0.04 & \\
\hline 5 & -7422.5119 & -7534.13 & -7496.5831 & $\omega_{5}$ & 1664.9132 & 1664.86 & 1664.8553 & 0.00 & \\
\hline 6 & -5851.1360 & -5962.91 & -5925.3547 & $\omega_{6}$ & 1571.3759 & 1571.22 & 1571.2284 & 0.01 & \\
\hline 7 & -4384.7147 & -4496.81 & -4459.1038 & $\omega_{7}$ & 1466.4213 & 1466.10 & 1466.2509 & 0.15 & \\
\hline 8 & -3041.1089 & -3153.45 & -3115.5628 & $\omega_{8}$ & 1343.6058 & 1343.36 & 1343.5410 & 0.18 & \\
\hline 9 & -1848.9391 & -1962.18 & -1923.3797 & $\omega_{9}$ & 1192.1698 & 1191.27 & 1192.1831 & 0.91 & \\
\hline 10 & -857.2981 & -972.95 & -931.9223 & $\omega_{10}$ & 991.641 & 989.23 & 991.4574 & 2.23 & $* * *$ \\
\hline 11 & -165.6714 & -279.32 & -236.4322 & $\omega_{11}$ & 691.6267 & 693.63 & 695.4901 & 1.86 & $*$ \\
\hline 12 & - & -27.71 & -3.6818 & $\omega_{12}$ & - & 251.61 & 232.7504 & 18.86 & $*$ \\
\hline
\end{tabular}

${ }^{9} \mathrm{BeD}$

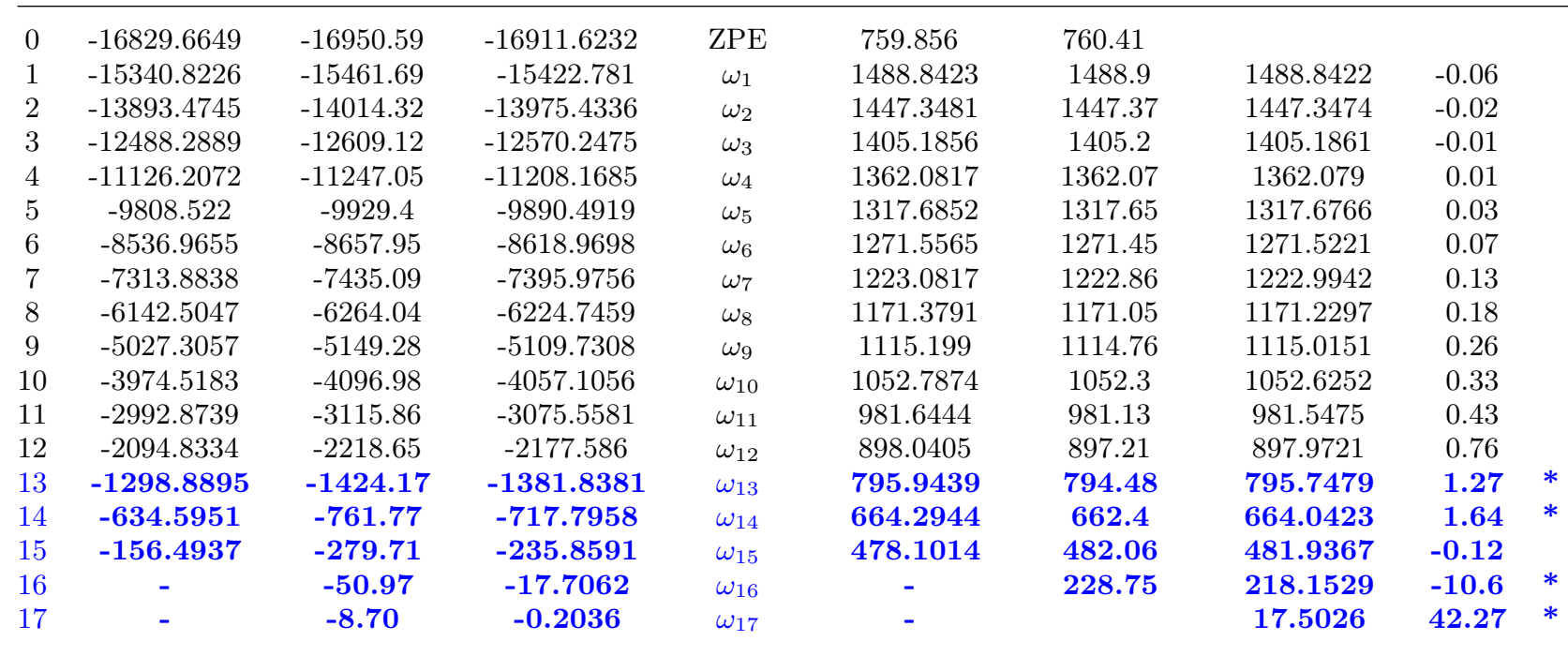

${ }^{9} \mathrm{BeT}$

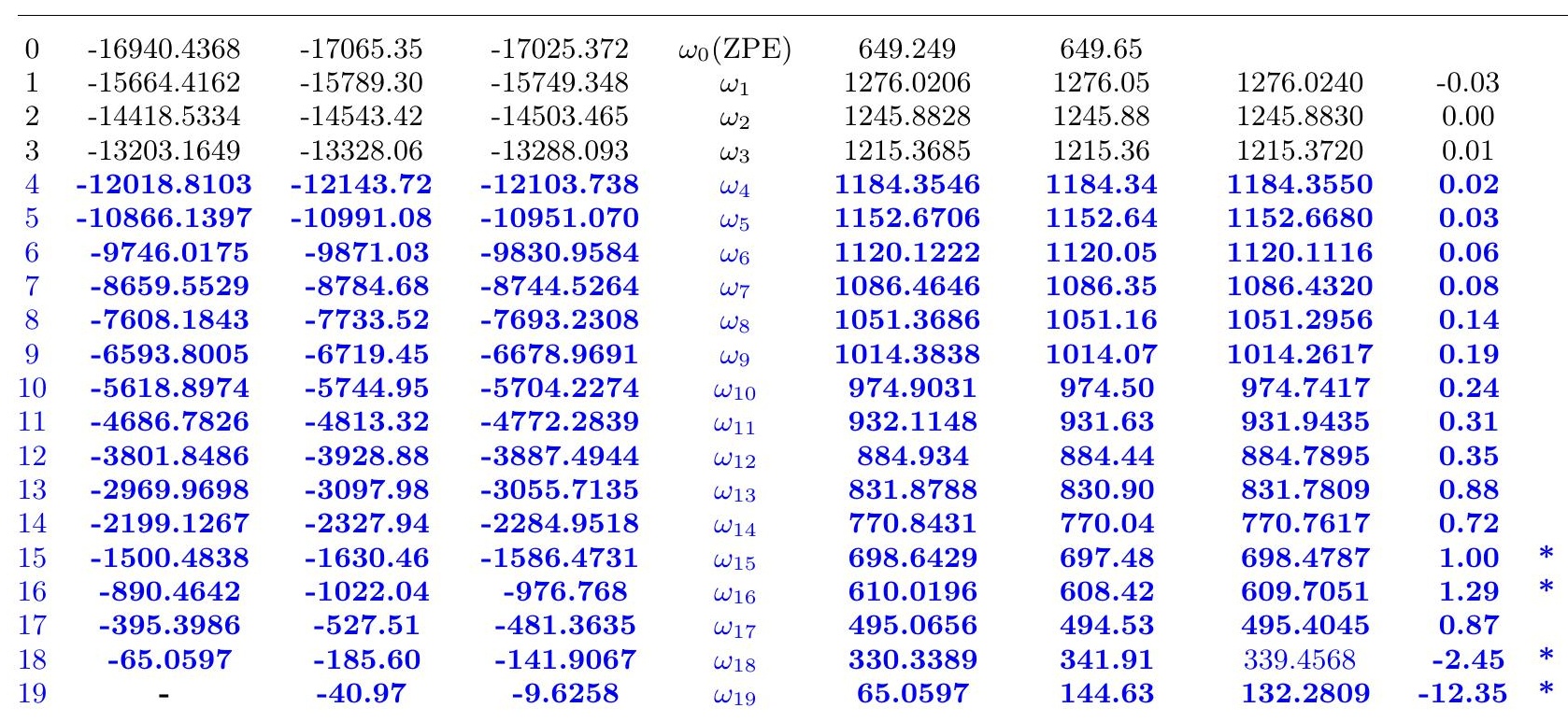

